\title{
Three-dimensional metallic fractals and their photonic crystal characteristics
}

\author{
Bo Hou, Hang Xie, Weijia Wen, and Ping Sheng \\ Department of Physics, The Hong Kong University of Science and Technology, Clear Water Bay, Kowloon, Hong Kong
}

(Received 18 September 2007; revised manuscript received 12 November 2007; published 11 March 2008)

\begin{abstract}
We report photonic properties of subwavelength three-dimensional (3D) metallic H-shaped fractals. The fractal structure supports localized resonances with relevant wavelength over ten times the sample size. Owing to the anisotropy inherent to the fractal geometry, the resonances and their induced band gaps are polarization dependent. The measured microwave transmission spectra agree well with simulations, and show the anisotropic response to be well described by an effective dielectric tensor. Using the three-dimensional $\mathrm{H}$ fractal as the basic unit, a microwave photonic crystal was fabricated, and its band-gap characteristics shown to display unique features of hybridization between local resonances and Bragg scattering. A photonic crystal of 3D fractals is an excellent microwave analog to an anisotropic electronic solid consisting of atoms or molecules with localized discrete energy levels.
\end{abstract}

DOI: 10.1103/PhysRevB.77.125113

PACS number(s): 42.70.Qs, 47.53.+n, 41.20.Jb, 78.90.+t

\section{INTRODUCTION}

The optical properties of materials are related not only to their crystal structures, but also to their atomic and molecular constituents. In the Lorentz model, the response of atoms and/or molecules to optical waves can be described in terms of electrons harmonically bound to ions, where the resonant frequencies correspond to the absorption peaks of the material. ${ }^{1}$ Generally, an atom's size is on the order of a few angstroms, much smaller than the wavelength of visible light, $\lambda \sim$ thousands of angstroms. In exact analogy to electronic systems, photonic crystals ${ }^{2}$ and metamaterials ${ }^{3,4}$ are artificial structures relying on periodicity and local resonances, respectively, for their unique characteristics. In particular, metamaterials owe their peculiar characteristics to resonant structural units, consisting of either conducting wires or split-ring resonators, ${ }^{3}$ that display subwavelength resonances similar to atoms and molecules, but at frequencies that are artificially tunable.

Light or electromagnetic wave interaction with fractals is an interesting topic because of the peculiar geometric scaling invariance of the structure. There is an extensive literature on optical properties of fractals formed from aggregation of metallic particles that are usually random in character. ${ }^{5-7}$ There have been a similar abundance of studies on the electrical properties of fractal-shaped electrodes or self-similar circuits. ${ }^{8-10}$ Electromagnetic functionalities of fractal patterns, such as Koch curves and Sierpinski gaskets, have been intensely investigated, e.g., for their applications as diffraction plates or radio antennas. ${ }^{11,12}$ Recently, electromagnetic (EM) wave localization has also been reported in a threedimensional fractal structure. ${ }^{13,14}$

In this work we present a multiresonance metamaterial structural unit in the form of a three-dimensional (3D) metallic $\mathrm{H}$-shaped fractal, operative in the microwave regime. This represents a 3D version of the two-dimensional (2D) $\mathrm{H}$ fractal. ${ }^{15,16}$ The additional spatial dimension means not only that the relevant resonances are further subwavelength than in the planar case, but also that there is a significantly richer array of characteristics that may be utilized for the control and manipulation of microwaves. After identifying the elec- tric and magnetic response of these 3D $\mathrm{H}$ fractals, we use them to construct photonic crystals and study their photonic characteristics. The photonic crystal made from these fractal units offers perhaps the most complex band structure yet observed in the microwave regime, combining the effects of local resonances, Bragg scattering, and anisotropy. Numerical simulation results are shown to be in excellent agreement with the experiment. In particular, our work is distinct from most previous works ${ }^{5-16}$ in its focus on the interaction of fractal local resonances with Bragg scattering.

\section{THREE-DIMENSIONAL H-SHAPED FRACTAL}

The starting point of our 3D fractal unit is a line of length $a$, defined as the first level of the structure, parallel to the $z$ axis. The $(k+1)$ th-level structure, containing $2^{k}$ lines, is constructed along the direction orthogonal to the plane formed by the lines of the previous two levels, with the midpoint of each line connected to the ends of the $k$ th-level lines. The length of the $(k+1)$ th-level lines is scaled as $a / 2^{\text {quo }(k, 3)}$, where the notation quo $(m, n)$ denotes the quotient of integer $m$ divided by integer $n$. Hence the first nine levels have line lengths $a$ for levels $1-3, a / 2$ for levels $4-6$, and $a / 4$ for levels 7-9. The construction process is illustrated in Fig. 1(a). The fractal dimension is given by $D_{f}$ $=\log _{10} 8 / \log _{10}(1 / 0.5)=3$, where 0.5 is the scaling factor and 8 is the number of self-similar units generated after scaling. ${ }^{17}$ This line structure therefore fills a $2 a \times 2 a \times 2 a$ cube as the number of levels, $N$, approaches infinity. It is interesting to note that the projections of the 3D fractal along the three orthogonal directions $x, y$, and $z$ are the planar $\mathrm{H}$ fractals. One such projection is shown in Fig. 1(b).

The experimental sample was a six-level 3D fractal with the first-level line length $a=25 \mathrm{~mm}$, made of metallic wires with a diameter of $0.9 \mathrm{~mm} .{ }^{18}$ A single unit is $4 \times 4 \times 4 \mathrm{~cm}^{3}$ in size. In order to increase the sample cross section so as to facilitate microwave measurements, a $7 \times 7$ square lattice comprising 49 3D fractal units was used, with a lattice constant of $6 \mathrm{~cm}$. Two identical horn antennas were connected with a network analyzer to generate and receive electromagnetic waves. The sample was placed between two antennas 

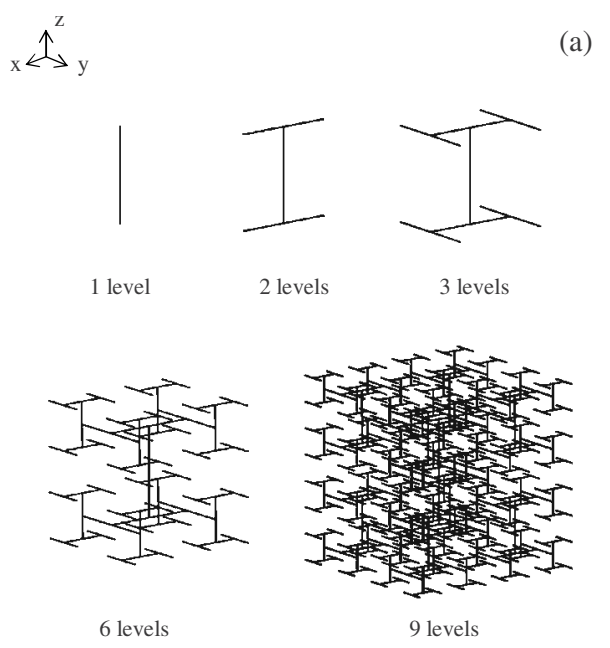

(b)

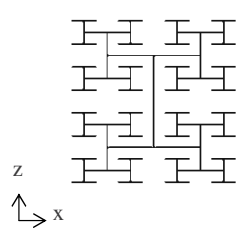

FIG. 1. (a) Iterative construction of the three-dimensional $\mathrm{H}$ fractals. (b) One of the projections of the nine-level 3D $\mathrm{H}$ fractal is exactly a six-level planar $\mathrm{H}$ fractal.

and the transmission spectra were detected at normal incidence, for frequencies in the range $0.7-18 \mathrm{GHz}$. All transmission data were normalized to the free space transmission value.

\section{TRANSMISSION MEASUREMENT}

Since the 3D fractal is anisotropic, the transmissions along three incident directions $(x, y$, and $z$ ) were measured. For each direction, the EM wave was incident normally on one face of the sample, with two polarizations measured by rotating the horn antennas $90^{\circ}$. The measurement geometries are schematically shown in Fig. 2(a) and the corresponding results shown in Fig. 2(b). One can obtain a total of six spectra for all incident directions and polarizations, but as long as the incident electric field ( $E$ field) is oriented along the same direction, the results are the same regardless of the illuminated face. Hence only one spectrum is shown in Fig. 2(b) for every orientation of the incident $E$ field. In order to explain the experimental observations, the finite-difference time-domain (FDTD) simulation was employed ${ }^{19}$ with periodic and perfect conductor boundary conditions. The simulation results are superposed on the corresponding experimental data as solid lines in Fig. 2(b). Good agreement between experiment and theory can be seen. The six-level fractal structure shows a total of six band gaps within the measurement frequency range, each corresponding to a local resonance (see below). Four bands have their wavelengths larger than $6 \mathrm{~cm}$, the lattice constant. In particular, the lowest-frequency resonance occurs at the relevant wave-

(a)

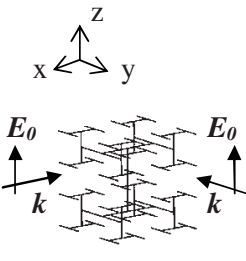

$E_{0} / / z$ axis

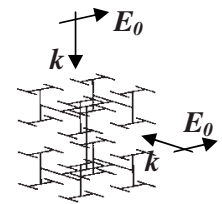

$E_{0} / / x$ axis

Wavelength $(\mathrm{cm})$

(b)

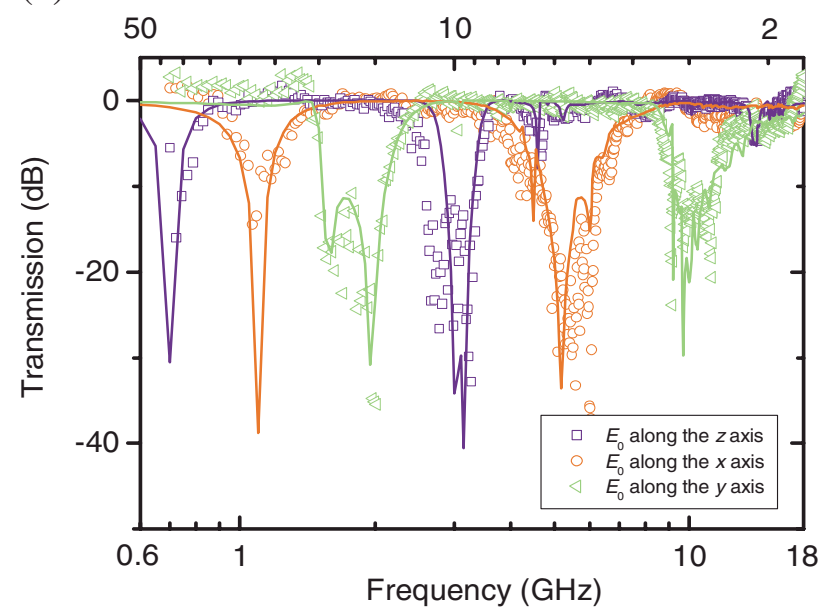

FIG. 2. (Color online) (a) Schematics of measuring the six-level fractal under various microwave illuminations, incident directions, and polarizations. $\boldsymbol{E}_{0}$ is the electric vector of the incident wave and $\boldsymbol{k}$ is the wave vector. The six-level fractal measures $4 \times 4 \times 4 \mathrm{~cm}^{3}$. Of the six measurement geometries, every two cases with the same incident polarization have the same spectra. (b) Transmission spectra of a $7 \times 7$ square array of the six-level fractals at normal incidence, for those cases as shown in (a). Open symbols denote the experimental values and the solid lines are the corresponding simulation results.

length of $\sim 41 \mathrm{~cm}$ (at a frequency of $0.7 \mathrm{GHz}$ ), which is more than ten times the edge length of the structure, indicating the subwavelength characteristic. Furthermore, the appearance of these transmission stop bands is dependent strongly on the polarization of the incoming EM radiation, which is the result of the geometrical anisotropy. We also measured the spectra at oblique incidence, four cases of which are shown in Fig. 3. For TE waves [shown in Fig. 3(a)], the spectra are nearly identical with each other because $\boldsymbol{E}_{0}$ is parallel to the first level line. However, for TM waves [shown in Fig. 3(b)], two new dips become apparent at lower frequencies as the incident angle increases, and their positions correspond to the 0.7 and $3.0 \mathrm{GHz}$ stop bands in Fig. 2 . This is due to the fact that $\boldsymbol{E}_{0}$ has more and more components projected along the first-level line as the wave vector $\boldsymbol{k}$ is rotated. Similar results for other oblique incidence cases were observed, and they imply that the six stop bands are the basic responses of the fractal interacting with EM waves. Photonic responses from arbitrary incident angles may be expressed as linear combinations of these six basic spectra.

The simulations reveal that the formation of the stop bands originates from a series of resonances intrinsic to the 

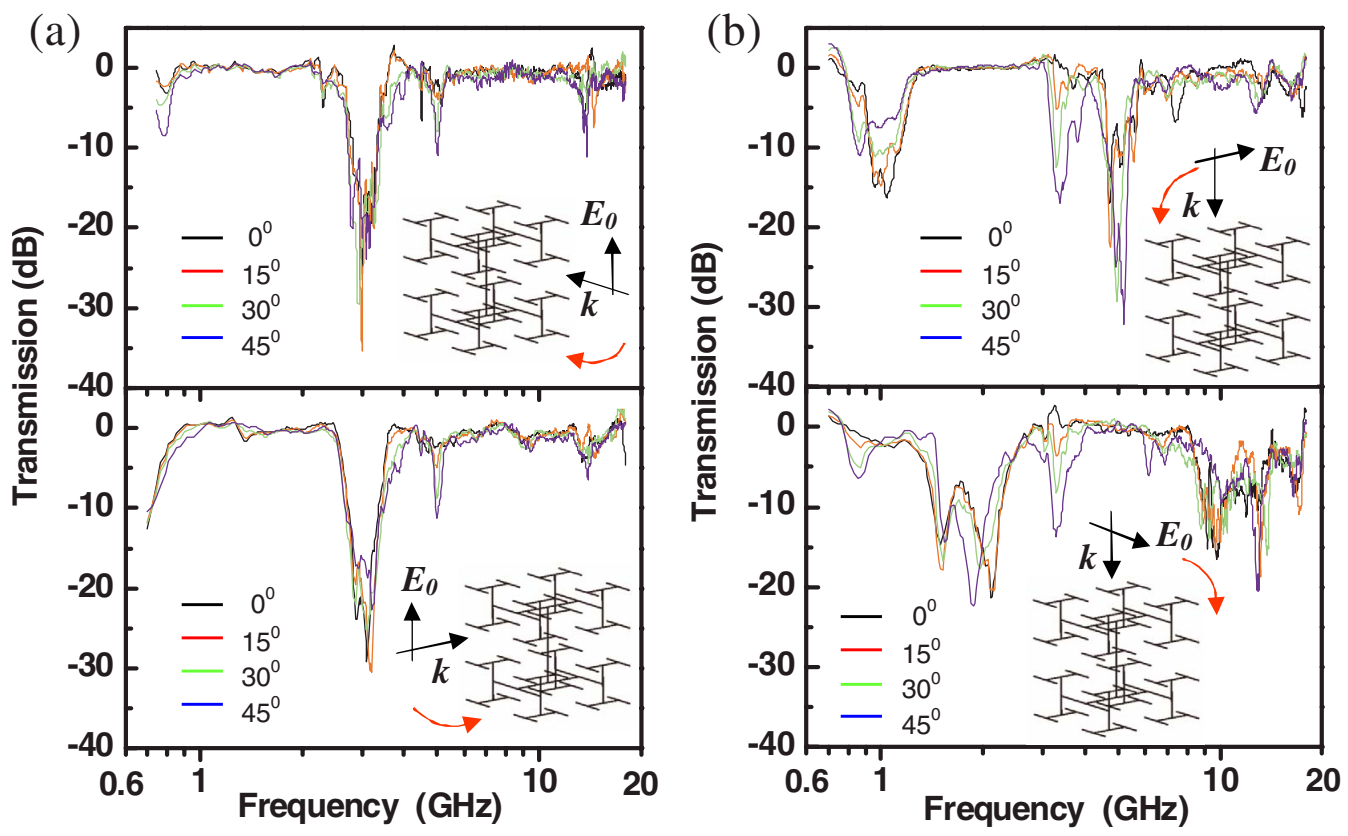

FIG. 3. (Color online) Transmission spectra of the square array under oblique incidence. The incidence geometries are illustrated in the insets. The incidence angles are $0^{\circ}, 15^{\circ}, 30^{\circ}$, and $45^{\circ}$. (a) The case of TE waves where the incident wave vector $\boldsymbol{k}$ was rotated within the plane perpendicular to the first level line. (b) The case of TM waves where $\boldsymbol{k}$ was rotated within the plane defined by the first level line and the $\boldsymbol{E}_{0}$ vector.

fractal structure, and these resonances are characterized by distinct current distributions. In Fig. 4 we plot the calculated surface current distributions on the fractal structure for every stop band frequency. ${ }^{20}$ It is seen that every resonance leads to a specific current distribution with intensity maximum located on some specific level. For the lowest-frequency resonance, defined as the fundamental mode, the current maximum appears only on the first-level line. As the frequency increases, the resonant current distribution shifts to higher levels. For the highest one, the maximum is on the sixthlevel lines.

\section{SUBWAVELENGTH CHARACTERISTICS}

We are particularly interested in the subwavelength feature of the fundamental mode, where the fractal structure is smaller than one-tenth of the relevant wavelength. The fractal includes a maximum conducting path $l$ extending from one corner of the structure along the body diagonal to the opposite corner, which is longer than the edge length of the fractal body. For a 3D H-shaped fractal with $N$ total levels, the maximal path length is given by

$$
l_{N}=\sum_{j=1}^{\mathrm{quo}(N, 3)} \frac{3 a}{2^{j-1}}+\sum_{j=1}^{\operatorname{rem}(N, 3)} \frac{a}{2^{\text {quo }(N, 3)}}= \begin{cases}3 a+\frac{3 a}{2}+\cdots+\frac{3 a}{2^{i-1}}=6 a-\frac{3 a}{2^{i-1}}, & \text { if } N=3 i, \\ 3 a+\frac{3 a}{2}+\cdots+\frac{3 a}{2^{i-1}}+\frac{a}{2^{i}}=6 a-\frac{5 a}{2^{i}}, & \text { if } N=3 i+1, \\ 3 a+\frac{3 a}{2}+\cdots+\frac{3 a}{2^{i-1}}+\frac{a}{2^{i}}+\frac{a}{2^{i}}=6 a-\frac{a}{2^{i-2}}, & \text { if } N=3 i+2,\end{cases}
$$

where $i$ and $j$ are positive integers, the notation $\operatorname{rem}(m, n)$ means the remainder of integer $m$ divided by $n$, and the summation should be neglected when the upper limit is less than the lower one. If $N=1$, then $l_{1}=a$. For a dipole (i.e., just a line of length $l_{1}$ ), the resonant wavelength of the fundamental mode $\lambda_{1}=2 l_{1} \cdot{ }^{21}$ When the lines of higher levels are added, as shown in Fig. 1(a), the path becomes longer (see the inset in Fig. 5) and the resonance wavelength is expected to increase. However, for the fractal this increase is faster than linear. We have simulated the values of $\lambda_{N}$ (the fundamental mode) while $N$ increases from 1 to 9 , and depict the variation of the resonance wavelength versus the conducting path in Fig. 5, where both quantities are normalized with respect to $\lambda_{1}$ and $l_{1}$, respectively. Clearly the variation (solid 


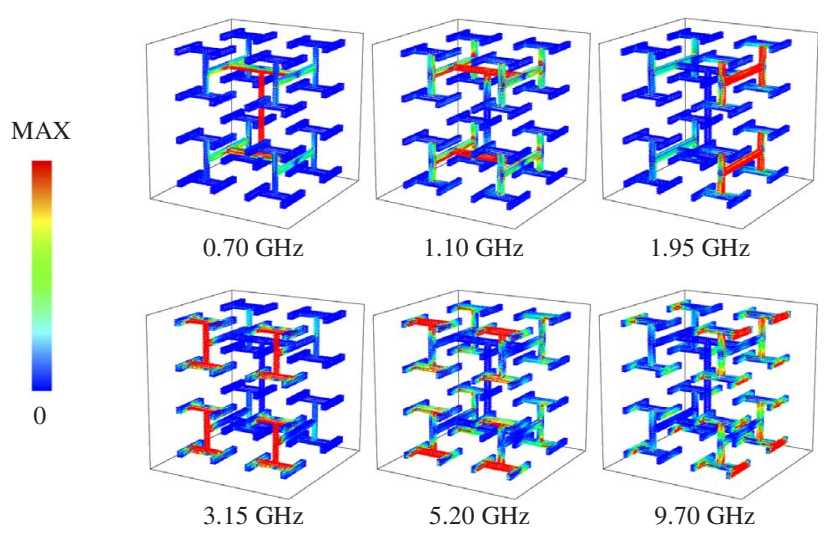

FIG. 4. (Color online) Simulated surface current distributions at the six band-gap frequencies denoted in the figure. These frequency values correspond to the minimum transmittance positions of the band gaps in the simulation.

squares) for the 3D fractal case is nonlinear and, as the levels increase, rises rapidly above the linear relationship $\lambda=2 l$ that is relevant to the dipole case. The upward deviation from the dipole case, as well as the increasing ratio of $\lambda_{N}$ to $l_{N}$, imply that the subwavelength feature of the fractal structure is more than just an increase in path length. The influence of higher levels (i.e., finer structure) on the fundamental resonance does play an important role. In other words, while the dominant (resonant) current distribution is centered at the level-1 line, the small tail of currents flowing into the higherlevel lines actually has a material effect in lowering the fundamental resonance frequency $f_{0}$. From the perspective of equivalent circuit modeling, the resonant frequency is given by $2 \pi f_{0}=1 / \sqrt{L C}$. The higher-level structures can also contribute to the capacitance $C$ and inductance $L$ of the equivalent circuit. ${ }^{21}$ Since the higher-level structures of a 3D H fractal are ramified and contain many curled lines within a limited spatial region, they can efficiently increase the capacitance $C$ and inductance $L$ for the entire structure.

For comparison, we also plot in Fig. 5 the same resonance wavelength variation (solid circles) for a planar $\mathrm{H}$ fractal with the levels increasing from 1 to 9 . Despite rising faster than in the $3 \mathrm{D}$ case, the curve for the planar $\mathrm{H}$ fractal case stops at a lower wavelength value, a direct consequence of the $2 \mathrm{D}$ geometry. This is in spite of the fact that a nine-level planar $\mathrm{H}$ fractal (five orders of scale $a, a / 2, a / 4, a / 8$, and $a / 16)$ has a larger edge length than its nine-level 3D counterpart. For the same edge length, in the planar case the level can increase up to 6 only [exactly the planar projection of the nine-level 3D H fractal; see Fig. 1(b)], and correspondingly $l_{N} / l_{1}$ stops at the value of 3.5. Hence it is seen that, for the same sample size, the 3D H fractal can be much more subwavelength than the $2 \mathrm{D}$ case.

There are other 3D space-filling curves, such as the Hilbert curve, which has the same fractal dimension as the $\mathrm{H}$ fractal. It was found that the 3D Hilbert curve of few levels can support very far subwavelength EM resonances owing to its efficient space-filling property. ${ }^{12}$ A detailed comparison between these two fractals, in particular with regard to the (saturated or divergent) behavior in the limit of $\lambda_{N}$ at $N$

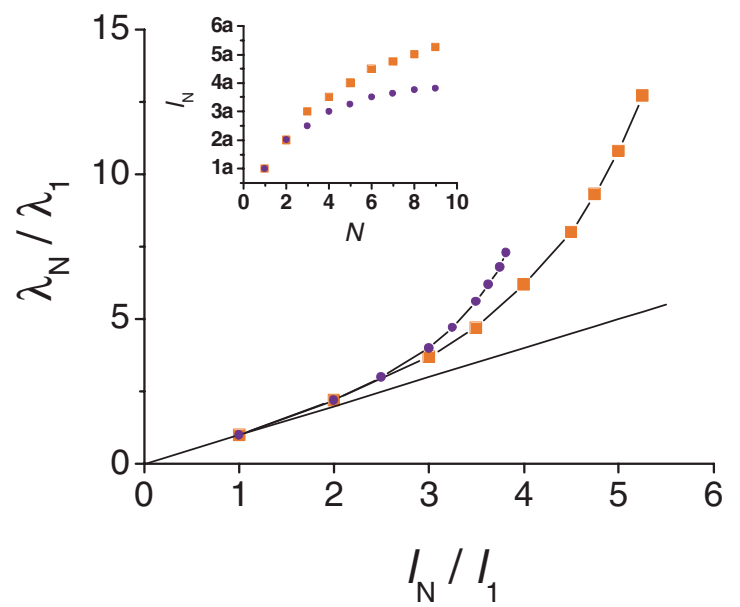

FIG. 5. (Color online) Variation of the resonance wavelength of the fundamental mode, $\lambda_{N}$, versus the maximal conducting path $l_{N}$ in an $\mathrm{H}$ fractal structure as the number of levels, $N$, is increased. The line with circles is for the planar $\mathrm{H}$ fractal and the line with squares is for the $3 \mathrm{D} \mathrm{H}$ fractal. Both the resonance wavelength and the path length have been normalized with respect to the values of the first-level structure $(25 \mathrm{~mm})$. The solid squares and circles denote the simulated values for the $3 \mathrm{D}$ and planar $\mathrm{H}$ fractals, respectively. The solid line indicates the dipole relationship $\lambda=2 l$. Inset: Plot of $l_{N}$ vs $N$ (from 1 to 9) for the 3D (solid squares) and planar (solid circles) $\mathrm{H}$ fractals. When $N \rightarrow \infty, l_{N} \rightarrow 6 a$ for the 3D case and $4 \mathrm{a}$ for the planar case.

$\rightarrow \infty$, should be interesting but is out of the scope of this paper.

\section{EFFECTIVE DIELECTRIC RESPONSE}

From Fig. 4 we see the distribution of the resonant current looks like the letter $\mathrm{H}$, owing to the H-shaped symmetry of the 3D fractal. Since the excitation of magnetic resonances in metamaterials requires a loop (or partial loop) distribution of the current in accordance with Faraday's law, ${ }^{22}$ we conclude that the present structure does not support magnetic excitations, at least in the long-wavelength limit. Thus, these resonances may be characterized by an effective electric polarizability tensor

$$
\bar{\alpha}=\left(\begin{array}{ccc}
\alpha_{x} & 0 & 0 \\
0 & \alpha_{y} & 0 \\
0 & 0 & \alpha_{z}
\end{array}\right),
$$

and the square lattice of fractals may be modeled by a homogeneous plate (assuming the same thickness) with an effective permittivity tensor

$$
\bar{\varepsilon}=\left(\begin{array}{ccc}
\varepsilon_{x} & 0 & 0 \\
0 & \varepsilon_{y} & 0 \\
0 & 0 & \varepsilon_{z}
\end{array}\right),
$$

where $\varepsilon_{x}, \varepsilon_{y}$, and $\varepsilon_{z}$ are dielectric functions with Lorentzian profiles. ${ }^{23}$ By fitting the transmission spectra, we obtain the forms of the three elements of $\bar{\varepsilon}$ : 

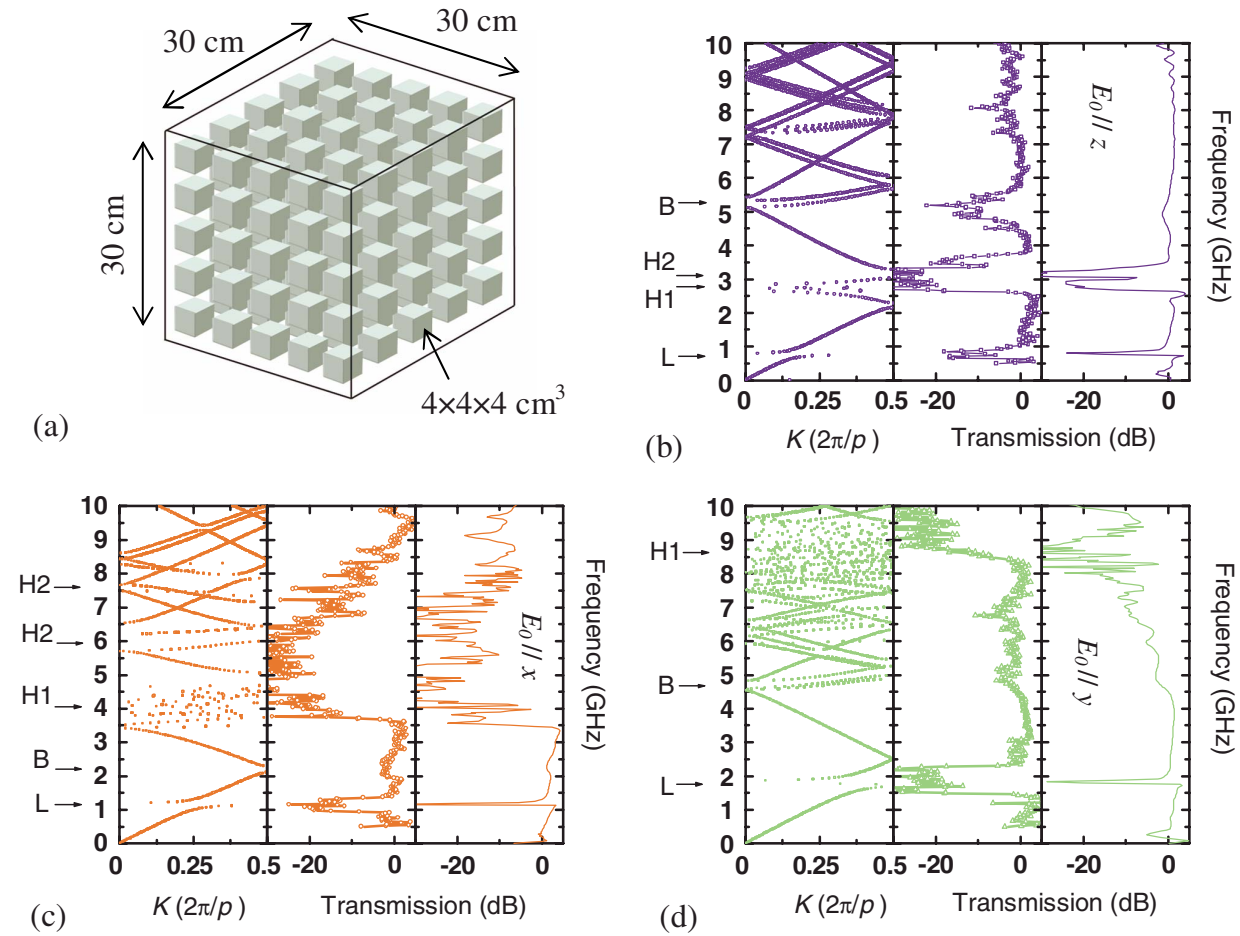

FIG. 6. (Color online) (a) Schematic representation of the $5 \times 5 \times 5$ photonic crystal of the six-level 3D fractals where each fractal unit is represented by a small cube with effective dielectric function given by Eqs. (3) and (4). The lattice constant for the simple cubic array is $p=6 \mathrm{~cm}$ and the side length for the small cube is $4 \mathrm{~cm}$. (b), (c), (d) Band structures (left) and transmission spectra measured (center) and simulated (right) at normal incidence for $\boldsymbol{E}_{0}$ aligned along the $z, x$, and $y$ axes, respectively. $K$ represents the wave vector along the incident direction. Those transmission values greater than 1 are due to wave diffraction and finite-size effects of the sample. The arrows, located beside the band diagrams and denoted $L, B$, and $H$, are to label the position of the local resonance, Bragg scattering, and their hybrid effect, respectively.

$$
\begin{aligned}
& \varepsilon_{x}=0.40+\frac{0.4}{1.15^{2}-f^{2}}+\frac{28.0}{4.60^{2}-f^{2}}, \\
& \varepsilon_{y}=0.40+\frac{1.0}{1.80^{2}-f^{2}}+\frac{80.10}{9.5^{2}-f^{2}}, \\
& \varepsilon_{z}=0.80+\frac{0.25}{0.72^{2}-f^{2}}+\frac{3.10}{2.75^{2}-f^{2}},
\end{aligned}
$$

where $f$ denotes the frequency (in $\mathrm{GHz}$ ), and the damping has been set to zero (since at microwave frequencies the metallic dissipation is negligible). The applicability of the expressions degrades at higher frequencies, where the permittivity is seen to be less than 1 . This is because the effective medium model works accurately only at low frequencies, where diffraction effects (from the local microstructures of the fractals) are absent. In addition, it is noted that the numerators in the Lorentzian expressions generally increase with increasing resonance frequency. This is because the number of lines supporting a specific local resonance increases with increasing resonance frequency (see Fig. 4). Hence there can be stronger coupling among the higherfrequency resonances, leading to a larger bandwidth. For example, in Fig. 2 the splits seen in the transmission dips at higher frequencies can be attributed to the coupling interac- tion. That directly corresponds to a larger numerator in the Lorentzian profile. Further investigations would be needed to clarify whether there is a scaling relation between the numerator values and resonance frequencies.

\section{PHOTONIC CRYSTAL OF FRACTALS}

When fractal units are arrayed periodically, the transmission spectrum displays not only the local resonances but also the Bragg scattering characteristics. Experimentally, we have fabricated a $5 \times 5 \times 5$ array of $3 \mathrm{D}$ fractals, arranged into a simple cubic lattice with lattice constant of $6 \mathrm{~cm}$ and supported by foam, with a dielectric constant $\sim 1$. This is schematically illustrated in Fig. 6(a). All fractal units are oriented identically. The normally incident transmissions were measured for EM waves illuminating different surfaces of the cube with two different polarizations. The measured spectra, within the frequency range of $0.5 \mathrm{GHz}$ to $10.0 \mathrm{GHz}$, are plotted in Figs. 6(b)-6(d). Just as in Fig. 2, only one spectrum is shown for every incident polarization direction, since the results are almost identical for different illumination surfaces.

We use the FDTD approach to simulate the transmission spectra of a $5 \times 5 \times 5$ photonic fractal crystal. For simplification, within each unit cell $\left(6 \times 6 \times 6 \mathrm{~cm}^{3}\right)$ a homogeneous cube $\left(4 \times 4 \times 4 \mathrm{~cm}^{3}\right)$ with the dispersive characteristics as 
(a)

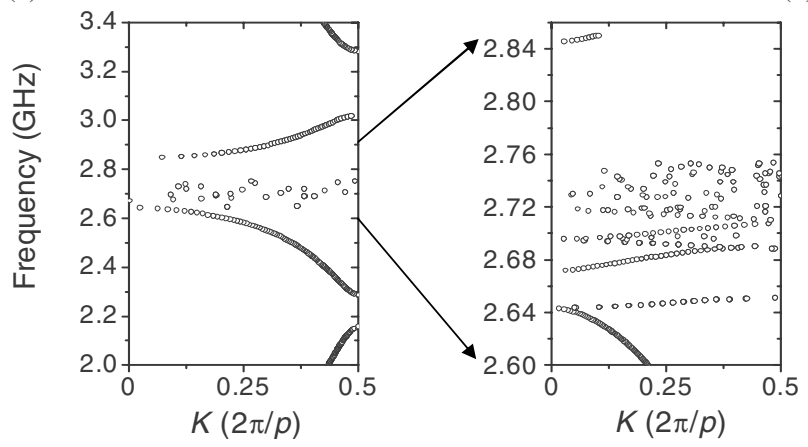

FIG. 7. (a) The same band structure as in Fig. 6(b), magnified within the frequency range 2.0-3.4 GHz. (b) The same band structure as in (a), but further magnified for the frequency range 2.60-2.86 GHz. The plurality of the folded structure is evident. This is a direct consequence of the very large dispersion of the fractal effective dielectric constant. The lattice constant $p=6 \mathrm{~cm}$.

given by Eq. (4) is used to stand for the real fractal. An incident pulse with a broad frequency spectrum is normally incident on the $5 \times 5 \times 5$ crystal array. The transmitted field is recorded behind the crystal. Perfect matched layer absorption boundary conditions are implemented. The simulated results are shown to the right of the measured transmission curves in Figs. 6(b)-6(d). The band structures of these effective dispersion crystals are also calculated by the finiteelement method, using the commercial software COMSOL. Periodic boundary conditions are assumed, and the results are shown to the left of the measured transmission curves in Figs. 6(b)-6(d).

From the band diagrams and the spectra, we see that local resonances (the atomic form factor), Bragg scattering (the structure factor), and their hybridizations (when the relevant frequencies of the two are close to each other) can all contribute to the transmission dips. We label these three effects respectively by $L, B$, and $H$ and point out, with arrows in the band-structure diagrams, the positions where they occur. We note that Bragg scatterings happen around 2.5 and $5 \mathrm{GHz}$ (when the lattice constant is $6 \mathrm{~cm}$ ), where small dips are observed in the transmission spectra. The dips from the Bragg scattering are small, since the gap exists only in the incident direction and hence does not constitute an absolute (or omnidirectional) band gap. Waves can therefore be transmitted through directions other than the incident direction and collected by the receiving horn. For the lowest three resonances, the characteristic of the band gaps caused by the local resonances is obvious in the band-structure diagrams, as indicated by the three $L$ arrows.

When the frequency of the local resonance, $f_{L}$, is not well separated from that of Bragg scattering, hybridization of the two occurs. There can be two types of hybridization, depending on whether the frequency is in the (large) positive dielectric constant side of the local resonance frequency $\left[f<f_{L}\right.$, denoted $H 1$ in Figs. 6(c) and 6(d)], or in the negative dielectric constant side $\left(f>f_{L}\right.$, denoted $\left.H 2\right)$. For the $H 1$ type, the dispersion curve of the small cube, $2 \pi f=c K / \sqrt{\varepsilon}$ ( $K$ being the wave vector and $c$ the speed of light in vacuum), becomes
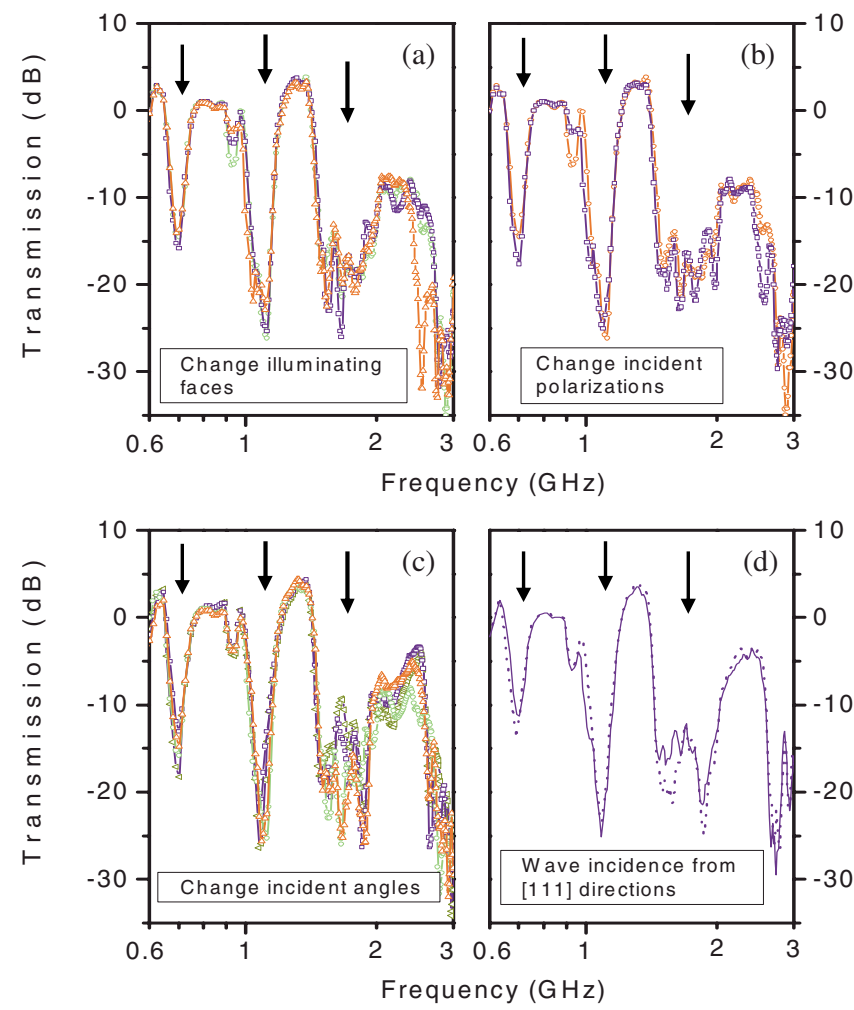

FIG. 8. (Color online) Measured transmission spectra of a 5 $\times 5 \times 5$ photonic crystal of the six-level 3D fractal structure, whose orientations are dispersed in three orthogonal directions, under various illumination conditions. (a) Microwaves were normally incident on three different faces of the array. (b) For one face, the polarization of the incident wave was changed. For the other faces, similar spectra were obtained and hence not shown. (c) For one face and $S$ polarization, the incident angle was changed from $0^{\circ}$ to $15^{\circ}, 30^{\circ}$, and $45^{\circ}$. For other polarizations and other faces, the spectra are the same and hence not shown. (d) The waves were incident from the [111] directions. Only two spectra are shown; the measurements for the two other diagonal directions produced the same results and hence are not shown. The arrows mark the three absolute band gaps. Transmission values greater than unity are due to diffraction and finite-size effects.

flatter and flatter as the frequency approaches the resonance frequency (a singularity in the dielectric constant) from the (large) positive dielectric constant side. At the same time, because of the lattice periodicity, the flat $f(K)$ curve would fold many times within the Brillouin zone, giving rise to a series of approximately parallel and closely separated branches. This can be seen clearly in Fig. 7, which gives the magnified band structure of the $H 1$ regime seen in Fig. 6(b), in the regime from 2.0 to $3.4 \mathrm{GHz}$. These states are difficult to excite, owing to the very narrow resonance width, and so appear as stop bands for the measured transmission spectra, within which there can be a few sharp peaks near the band edges (see the simulated spectra). For the $H 2$ type, there exist some well-separated bands within the frequency region $f>f_{L}$ where the dielectric constant of the small cube is negative, as indicated by the $H 2$ arrows in Figs. 6(b) and 6(c). A negative dielectric constant implies exponentially decaying waves and would therefore prohibit the existence of photonic 
states inside the bulk material. However, since only $\sim 30 \%$ of the photonic crystal volume is occupied by the cubes, energy bands are allowed within the frequency regime of negative permittivity, formed by strong Bragg scattering. ${ }^{24}$ These discrete energy bands can also give rise to transmission peaks inside the band-gap frequency, as evidenced by the $H 2$ arrow in Fig. 6(b) (around $3 \mathrm{GHz}$ ).

Thus, treating the fractal as a unit with an associated effective permittivity tensor can yield good agreement between theory and experiment. Hybridization between the local resonances of the basic structural unit and lattice periodicity is seen to give rise to some fairly unusual characteristics. ${ }^{24-29}$

Since the fractal units are anisotropic, we have also studied the effect of orienting the fractal units along different directions, by tuning the orientations of each unit by $90^{\circ}$ relative to its neighbors, so that every orientation within the finite-sized sample has almost equal representation along the three orthogonal directions [100], [010], and [001]. The measured transmission spectra are shown in Fig. 8. Three band gaps are identified which are independent of the incident direction, angle, and polarization of the waves. They are hence absolute band gaps. A particularly striking characteristic of these band gaps is the flat band edges, owing to the local resonance origin of the gaps. By comparing the band gap positions in Figs. 2 and 8, the three absolute band gaps are seen to originate from the three lowest resonances of the fractal unit. While each fractal unit is anisotropic, the array is now isotropic in the long-wavelength regime. The reason lies in the subwavelength feature of localized EM resonances. Inside the cubic array, three fractal units oriented in three different direction can be regarded as an isotropic "superunit" which is still smaller than the relevant wave- length and hence below the diffraction limit. Yet they simultaneously display the three lowest resonances. Therefore, the array or the collection of such superunits appears isotropic at lower frequencies, with three absolute band gaps which originate from localized resonances.

\section{CONCLUDING REMARKS}

By fabricating a metallic 3D H fractal wire structure and measuring its transmittance, we show that the 3D fractal displays subwavelength resonances that are polarization and incident-angle dependent. A photonic crystal composed of such fractal units was made; it displays features in excellent agreement with those predicted by using the anisotropic effective dielectric function. With the help of band-structure calculations, the hybridization effect resulting from the strong interaction between local resonances, inherent to the fractal unit, and Bragg scattering is detailed. We also show the creation of absolute band gaps by means of random orientation of the fractal units. Possible applications include using the 3D fractal in subwavelength antenna design, a subwavelength resonant cavity using an inverted 3D fractal structure, and wave filtering, guiding, and slowing by a photonic crystal made of fractal units.

\section{ACKNOWLEDGMENTS}

We wish to acknowledge partial support for this work by Hong Kong RGC Research Grant No. HKUST 3/06C. H.X. would like to thank Dezhuan Han for useful discussions and help on the FDTD simulation.
${ }^{1}$ J. D. Jackson, Classical Electrodynamics, 3rd ed. (Wiley, New York, 1999).

${ }^{2}$ J. D. Joannopoulos, P. R. Villeneuve, and S. Fan, Nature (London) 386, 143 (1997), and references therein.

${ }^{3}$ D. R. Smith, J. B. Pendry, and M. C. K. Wiltshire, Science 305, 788 (2004), and references therein.

${ }^{4}$ P. Sheng, Science 313, 1399 (2006); J. Pendry, Nat. Mater. 5, 599 (2006).

${ }^{5}$ V. A. Markel, L. S. Muratov, M. I. Stockman, and T. F. George, Phys. Rev. B 43, 8183 (1991).

${ }^{6}$ F. Brouers, D. Rauw, J. P. Clerc, and G. Giraud, Phys. Rev. B 49, 14582 (1994).

${ }^{7}$ S. V. Karpov, V. S. Gerasimov, I. L. Isaev, O. P. Podavalova, and V. V. Slabko, Colloid J. 69, 159 (2007).

${ }^{8}$ L. Nyikos and T. Pajkossy, Electrochim. Acta 30, 1533 (1985).

${ }^{9}$ B. Sapoval, R. Gutfraind, P. Meakin, M. Keddam, and H. Takenouti, Phys. Rev. E 48, 3333 (1993).

${ }^{10}$ R. M. Hill, L. A. Dissado, and R. R. Nigmatullin, J. Phys.: Condens. Matter 3, 9773 (1991).

${ }^{11}$ D. H. Werner and R. Mittra, Frontiers in Electromagnetics (IEEE, New York, 2000), and references therein.

${ }^{12}$ K. J. Vinoy, K. A. Jose, V. K. Varadan, and V. V. Varadan, Mi- crowave Opt. Technol. Lett. 29, 215 (2001); J. Romeu and S. Blanch, in Proceeding Digest of 2002 IEEE Antennas and Propagation Society International Symposium, San Antonio, 2002, Vol. 4, p. 550; J. McVay, N. Engheta, and A. Hoorfar, in Proceeding Digest of 2006 IEEE Antennas and Propagation Society International Symposium, Albuquerque, 2006, Vol. 1, p. 769.

${ }^{13}$ M. W. Takeda, S. Kirihara, Y. Miyamoto, K. Sakoda, and K. Honda, Phys. Rev. Lett. 92, 093902 (2004).

${ }^{14}$ K. Sakoda, Phys. Rev. B 72, 184201 (2005); Opt. Express 15, 1783 (2007).

${ }^{15}$ W. Wen, L. Zhou, J. Li, W. Ge, C. T. Chan, and P. Sheng, Phys. Rev. Lett. 89, 223901 (2002).

${ }^{16}$ L. Zhou, W. Wen, C. T. Chan, and P. Sheng, Appl. Phys. Lett. 82, 1012 (2003).

${ }^{17}$ See, e.g., Michael Barnsley, Fractal Everywhere (Academic Press, San Diego, 1988).

${ }^{18}$ The differences between the experimental sample and a true fractal in the mathematical sense are the finite diameters of the lines and the finite orders of scale. For our sample, there are only two orders of scale, $a$ and $a / 2$, inside the geometry.

${ }^{19}$ Simulations were performed using the software CONCERTO 3.5 (Vector Fields Ltd., England, 2004). 
${ }^{20}$ We obtained the surface current distributions $\left(I_{s}\right)$ by introducing a tiny loss into the perfect conductors and then extracting the spatial distributions of power dissipation $\left(P_{d}\right)$ on the conducting rods of the fractal at the resonance frequencies, according to the relation $P_{d}=I_{s}^{2} / Z_{s}$, where $Z_{s}$ is the surface impedance for a good conductor.

${ }^{21}$ B. A. Munk, Frequency Selective Surfaces: Theory and Design (Wiley, New York, 2000).

${ }^{22}$ W. J. Padilla, D. N. Basov, and D. R. Smith, Mater. Today 9, 28 (2006).

${ }^{23}$ C. F. Bohren and D. R. Huffman, Absorption and Scattering of Light by Small Particles (Wiley, New York, 1983).
${ }^{24}$ W. Y. Zhang, X. Y. Lei, Z. L. Wang, D. G. Zheng, W. Y. Tam, C. T. Chan, and P. Sheng, Phys. Rev. Lett. 84, 2853 (2000).

${ }^{25}$ D. F. Sievenpiper, E. Yablonovitch, J. N. Winn, S. Fan, P. R. Villeneuve, and J. D. Joannopoulos, Phys. Rev. Lett. 80, 2829 (1998).

${ }^{26}$ Z. Y. Li, B. Y. Gu, and G. Z. Yang, Phys. Rev. Lett. 81, 2574 (1998).

${ }^{27}$ A. Moroz, Phys. Rev. Lett. 83, 5274 (1999).

${ }^{28}$ Z. Liu, C. T. Chan, and Ping Sheng, Phys. Rev. B 65, 165116 (2002).

${ }^{29}$ B. Hou, J. Mei, M. Ke, W. Wen, Z. Liu, J. Shi, and P. Sheng, Phys. Rev. B 76, 054303 (2007). 